\title{
DC-Bus Signaling: A Distributed Control Strategy for a Hybrid Renewable Nanogrid
}

\author{
John Schönberger, Student Member, IEEE, Richard Duke, Member, IEEE, and \\ Simon D. Round, Senior Member, IEEE
}

\begin{abstract}
A de nanogrid is a hybrid renewable system since renewable sources supply the average load demand, while storage and nonrenewable generation maintain the power balance in the presence of the stochastic renewable sources. The system is power electronic based, with converters being used to interface both the sources and loads to the system. The nanogrid is controlled using dc-bus signaling (DBS), a distributed control strategy in which the control nodes, the source/storage interface converters, induce voltage-level changes to communicate with the other control nodes. This paper explains the control structure required for the converters to permit the use of DBS, and explains a procedure for implementing a system-wide control law through independent control of the source/storage interface converters. Experimental results are presented to demonstrate the operation of this novel control strategy.
\end{abstract}

Index Terms-DC system, distributed control, hybrid system, renewable system.

\section{INTRODUCTION}

D ISTRIBUTED generation using renewable sources is gaining popularity due to environmental concerns over burning fossil fuels, deregulation of the electricity industry, and technological advances in power electronics and renewable energy systems. Distributed renewable sources can be harnessed by using power-electronic converters to link them directly with the existing power system, or by combining them with local loads to form an independent power system [1]. A nanogrid falls into this category.

In this paper, the term nanogrid is used to describe a hybrid power system that supplies a cluster of loads with a peak rating in the order of 2-20 kW, since small-scale renewable generation tends to be more expensive than conventional forms of generation, nanogrids are mainly suited for niche applications such as power supplies for remote isolated locations. Constructing a standalone system to supply a cluster of remote loads is often cheaper than connecting the remote loads to the conventional ac system.

The structure of a standalone hybrid renewable nanogrid is shown in Fig. 1. The building blocks of the nanogrid are powerelectronic interface converters. Step-up converters allow low

Manuscript received November 26, 2004; revised May 10, 2005. Abstract published on the Internet July 14, 2006.

J. Schönberger was with the Department of Electrical and Computer Engineering, University of Canterbury, Christchurch 8020, New Zealand. He is now with the Power Electronic Systems Laboratory, Swiss Federal Institute of Technology, 8092 Zürich, Switzerland (e-mail: schoenberger@lem.ee.ethz.ch).

R. Duke is with the Department of Electrical and Computer Engineering, University of Canterbury, Christchurch 8020, New Zealand.

S. D. Round is with the Power Electronic Systems Laboratory, Swiss Federal Institute of Technology, 8092 Zürich, Switzerland.

Digital Object Identifier 10.1109/TIE.2006.882012 voltage sources to discharge into the nanogrid, and step-down converters allow the loads to draw power from the nanogrid. A bidirectional converter allows the storage node to charge from and discharge into the nanogrid. The nanogrid operates at dc since dc offers advantages such as improved transmission efficiency and ease of interfacing asynchronous sources such as wind turbines to the system [2].

In standalone hybrid renewable systems, uncontrollable renewable sources supply the average load demand, while storage acts as an energy buffer, balancing short-term differences between the source and load powers. Nonrenewable generation is included to improve system reliability in the event of a longterm shortage of renewable energy.

To maximize the use of the renewable sources, the system must be controlled in an optimal fashion according to a system control law. The conventional means of achieving this objective is to use a central controller that is aware of each component in the system [3], [4]. However, the reliability of the system is degraded as a practical implementation of this control strategy creates dependence on a central controller and communication link.

To improve the system reliability while allowing the implementation of a control law, a distributed control strategy is preferred, as the system becomes independent of the central controller. DC-bus signaling (DBS) is a means of controlling a hybrid renewable system in a distributed fashion. This control strategy maintains the reliability inherent in the structure of the system by using the dc bus itself as the communication link.

DBS is an extension of the concept of using charge/discharge thresholds to schedule individual sources in a distributed fashion [5]. Scheduling of the sources is achieved by making the operation of the source/storage interface converters dependent on the level of the dc bus in relation to their charge/discharge threshold; however, voltage droop is included to allow multiple converters to operate at each threshold [6]. Voltage-level changes on the dc bus, which occur as changes in the system, force these converters to switch between constant voltage and constant power operation. These voltage-level changes on the dc bus are used by the source and storage interface converters to determine their mode of operation.

DBS is a low-cost strategy that is implemented by setting the voltage threshold at which each source/storage interface converter becomes active. This paper explains the mechanism by which DBS operates and outlines the control structure required for the source/storage interface converters to permit the implementation of DBS in a nanogrid. A procedure for implementing a system control law is given, and a prototype system based on a case study is presented to demonstrate the 


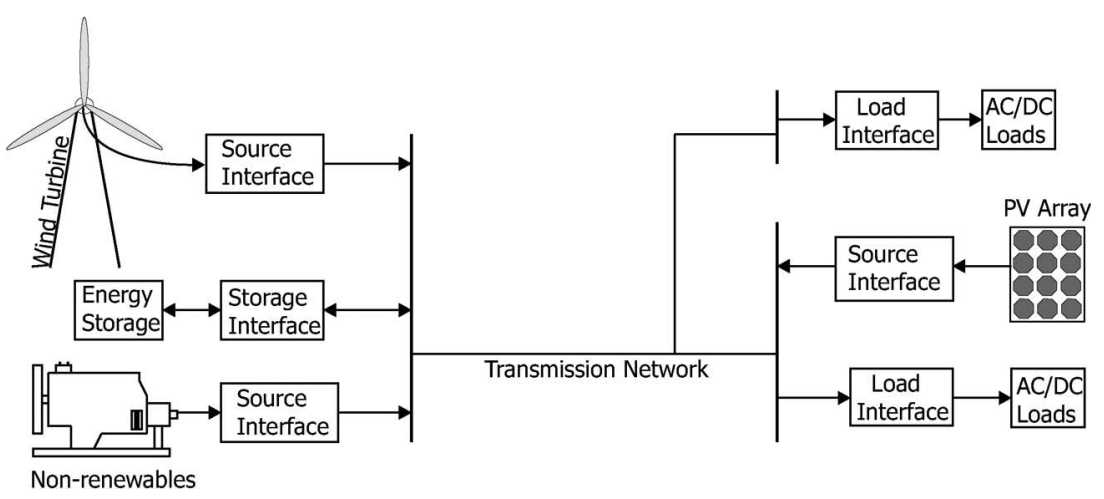

Fig. 1. Structure of a stand-alone hybrid renewable nanogrid.

practicality of the DBS in a system comprising renewable sources, battery storage, and backup generation.

\section{DBS}

The sources in a nanogrid can be controlled in a centralized, decentralized, or distributed fashion. With centralized control, each source is controlled from a single point using a central controller and communication link. A control law can be implemented easily since the central controller is aware of each node in the system, but the reliability of the system is degraded since the system depends on the communication link and controller for correct operation.

On the other hand, with decentralized control, each source operates independently using terminal quantities. Thus, the reliability inherent in the structure of a nanogrid is maintained. The drawback is that, implementing a control law to operate the system in an optimal fashion is impossible, since each node is unaware of the other nodes in the system.

With distributed control, the control function is distributed throughout the network. This strategy improves the reliability of the system over centralized control, since the system can still function if a node fails. Nevertheless, the system is dependent on an external communication link for correct operation.

The control strategy for a nanogrid should ideally maintain the advantages present in the distributed structure of the system while permitting implementation of a control law. None of the above control strategies can achieve both of these goals. Therefore, a hybrid distributed control strategy, DBS, is adopted in a bid to allow the implementation of a control law without the dependence on an external communication link.

As with distributed control, the control function for DBS is distributed among the source controllers that are located throughout the system. However, since the communication between the source controllers takes place over the dc bus rather than an external communication link, the sources are effectively controlled using terminal quantities, as with decentralized control. Thus, the DBS allows the implementation of a distributed control scheme with the same reliability advantages as decentralized control.

Control based on the voltage level of the dc bus has also been proposed to ensure high-priority loads in dc systems enjoy an uninterrupted supply of power under overload conditions [7], [8]. Prioritized load shedding is performed under overload con- ditions by controlling the loads to shut down at different voltage levels as the bus voltage collapses due to the overload. The main difference between the load shedding based on the voltage level of the dc bus and DBS is that DBS uses the dc-bus voltage for source scheduling, not load shedding. With DBS, the source and storage interface converters operate autonomously based on the voltage level of the dc bus. Each converter is assigned to a voltage threshold to trigger the point at which it begins discharging or charging.

The converters not only respond to the level of the dc bus, but they also change the level of the dc bus, automatically controlling other converters in the system. This autonomous operation is achieved by controlling the converters to exhibit three modes of operation: off, constant voltage, and constant power. Load changes and variations in generator output cause the interface converters to switch between constant voltage and constant power operation, changing the level of the dc bus. The utilization priority of each source is therefore dependent on the discharge/charge thresholds, hence, a control law is implemented by prioritizing these thresholds.

The use of DBS to schedule interface converters supplying power to a nanogrid, or discharging, is illustrated in Fig. 2. The example system, portrayed in Fig. 2(a), comprises a renewable source, a nonrenewable source, and a constant power load. The control law for the system has two operating states to prioritize the utilization of these sources. In state 1, only the renewable source is online, and in state 2 , the nonrenewable source comes online. The control law is implemented by assigning the renewable source to discharge at threshold $V_{0}$, and the nonrenewable source to discharge at threshold $\mathrm{V} 1$, as shown in Fig. 2(b).

When the load is less than the maximum power point (MPP) of the renewable source, PS1, the renewable source interface converter alone is online. The converter operates in a constant voltage mode, regulating the bus voltage to its discharge threshold, $V_{0}$. The bus voltage decreases slightly as the load current increases due to the voltage-droop characteristic of the converter. Thus, with a load of PL1, the system operates at point A in Fig. 2(b).

When the load exceeds the MPP of the renewable source, increasing to PL2, the renewable source interface converter operates in a constant power mode, limiting its output power to PS1. The bus voltage tends to collapse as the constant power load attempts to consume more power than the system can 


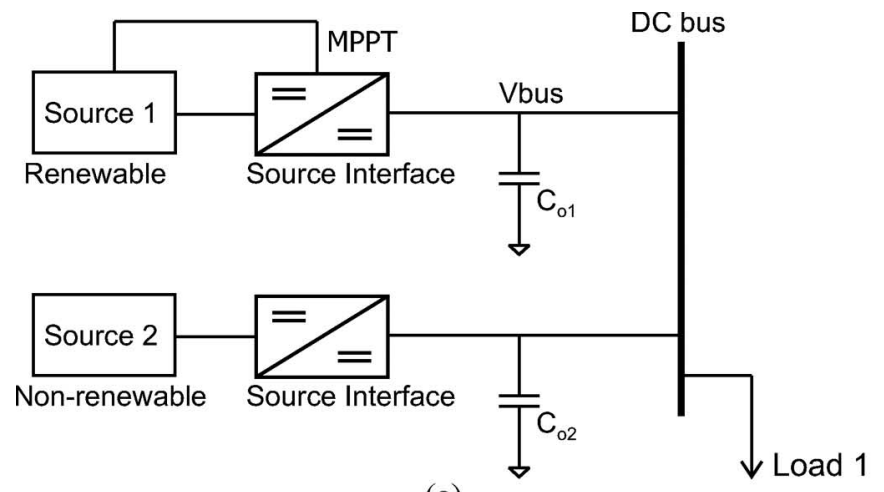

(a)

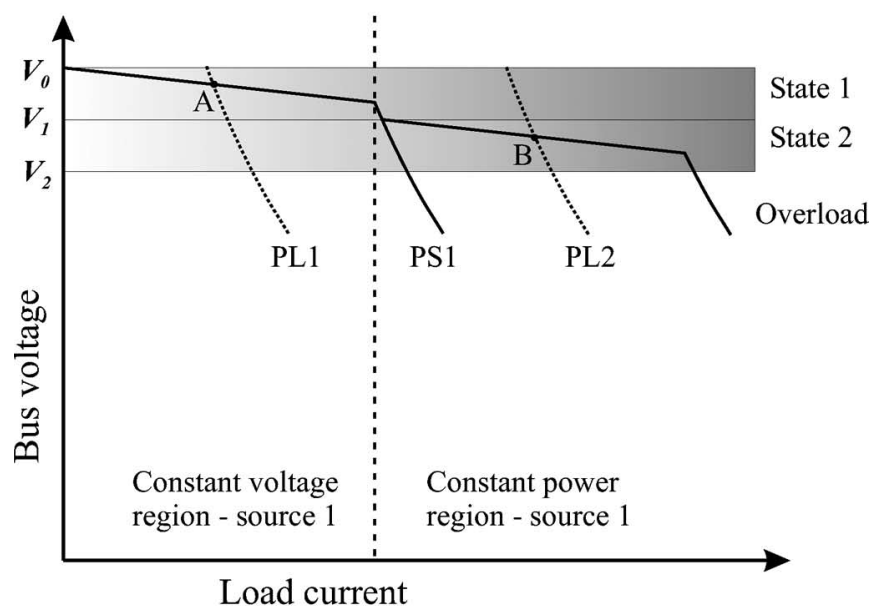

(b)

Fig. 2. Discharge scheduling using DBS. (a) System with discharge scheduling. (b) DBS operation-discharge.

provide. The converters' output capacitors temporarily support the dc bus; therefore, the decrease in the bus voltage is given by

$$
V_{\mathrm{bus}}(t)=\sqrt{\frac{2}{C_{\mathrm{bus}}} \int_{0}^{t}\left(P_{\mathrm{S} 1}(t)-P_{\mathrm{L} 2}(t)\right) d t} .
$$

When the bus voltage decreases to discharge threshold V1, the nonrenewable source interface converter comes online to supply the balance of power. The system now operates at point B.

The use of DBS to schedule the charging action of multiple storage interface converters is accomplished in a similar fashion. Since charging tends to collapse the dc bus, the storage node with the highest priority is assigned to the lowest voltage state. The storage interface converters charge while the bus voltage is above their charge threshold, and turn off when the bus voltage decreases below their charge threshold.

\section{Converter Control Structure}

To permit the use of DBS in a nanogrid, the source/storage interface converters are controlled to exhibit different modes of operation when discharging or charging. The operational mode of each converter is based on the voltage level of the dc bus in relation to its discharge/charge threshold.

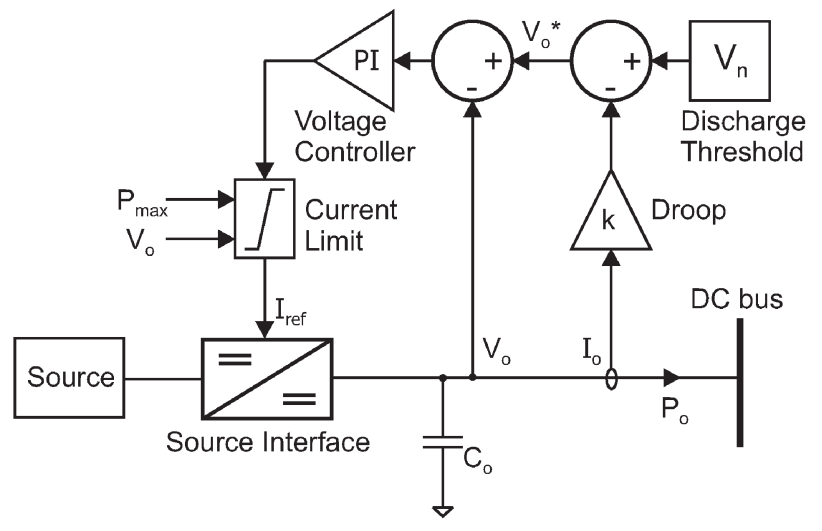

Fig. 3. Discharge controller.

\section{A. Discharge Controller}

The source/storage interface converters are designed with three modes of operation when discharging: off, constant voltage, and constant power. Each converter remains off until the bus voltage decreases below its discharge threshold. At this point, the converter comes online, acting as a constant voltage source. Once discharging, if the output power of the source interface exceeds the maximum power output of the source, the converter limits its output power.

The discharge control structure required to implement these modes of operation is shown in Fig. 3. The control structure is two tiered, comprising a fast inner current control loop and a slower outer PI voltage control loop. A dynamic current limit is included between the voltage and current loops. For simplicity, the current loop is not shown since its bandwidth is assumed to be an order of magnitude faster than the bandwidth of the outer control loop.

In constant voltage mode, the PI controller regulates the output voltage of the converter to its discharge threshold by providing a reference current to the inner loop. A voltagedroop characteristic is included in this mode to permit the load current sharing between other source/storage interface converters assigned to the same discharge threshold. The set point for the output voltage is given by

$$
V_{o}^{*}=V_{n}-k \cdot I_{o}
$$

where $V_{n}$ is the discharge threshold of the converter, $I_{o}$ is the output current of the converter, and $k$ is the droop coefficient.

The dynamic current limit is used to implement off and constant power modes. When the bus voltage is above the discharge threshold, the converter must be off, as converters with a higher utilization priority are capable of supplying the load. Under these conditions, the PI voltage controller attempts to provide a negative reference current to the inner loop. Therefore, imposing a lower limit of zero on the reference current deactivates the converter when the bus voltage is above its discharge threshold.

Constant power mode is implemented by dynamically adjusting the upper limit for the reference current to

$$
I_{\text {ref }}=P_{\max } / V_{o}
$$




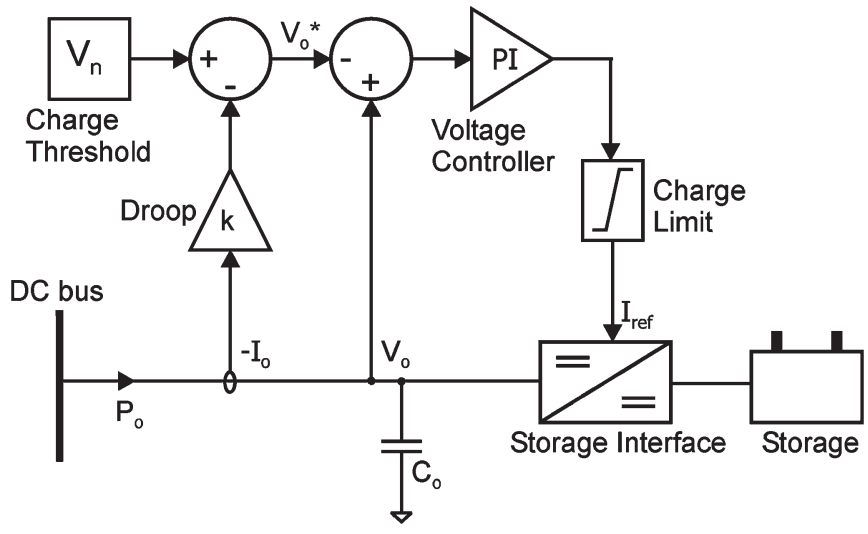

Fig. 4. Charge controller.

where $P_{\max }$ is the MPP of the renewable source or the peak discharge rating of the storage device. Thus, as $P_{\max }$ or the bus voltage changes, the reference current changes, such that the converter tracks $P_{\max }$.

\section{B. Charge Controller}

The storage interface converter is bidirectional, allowing the storage device to both discharge and charge. The storage interface therefore has two control structures, one for discharging and another for charging. The control structure used to implement the charging modes of operation is shown in Fig. 4.

When charging, a storage interface converter exhibits three modes of operation as with discharging: off, constant voltage, and charge limit. However, the operational modes are triggered by a rising bus voltage rather than a falling bus voltage, since charging tends to collapse the bus rather than support it.

The converter remains off while the bus voltage is below its charge threshold. When the bus voltage rises above this threshold, indicating excess renewable power in the system, the converter begins charging the storage device by operating in constant voltage mode. In constant voltage mode, the storage interface converter draws power from the system in a bid to regulate the dc bus at its charge threshold. The PI controller regulates the bus voltage to

$$
V_{o}^{*}=V_{n}+k \cdot I_{o}
$$

where $V_{n}$ is the charge threshold of the converter and $k$ is the droop coefficient that permits multiple storage nodes to share the total charging current. The droop relationship in (4) causes the bus voltage to increase as the charging current increases, because $I_{o}$ is negative under charging conditions.

In constant voltage mode, the storage interface converter operates as a slack bus because it charges using any excess renewable power, provided that its charge current limit is not exceeded. A current limit is imposed on the reference charge current to prevent the storage device from charging at a dangerously high rate when the excess renewable energy exceeds the charge rating of the storage device. This mode of operation is known as charge limit.

It should be noted that using the state of the dc bus alone to determine the operation of the storage node may degrade the lifetime of the storage device if it is a battery bank. With the

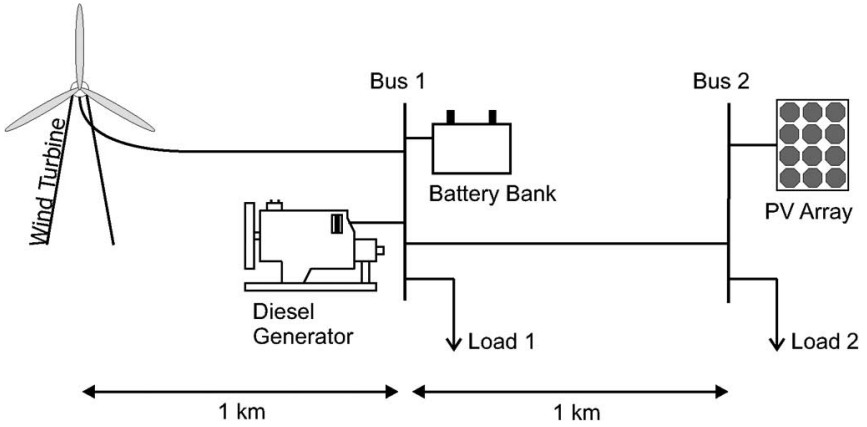

Fig. 5. Case study.

control structure shown in Fig. 4, all excess power from the renewable sources is used to charge the storage device, and the batteries are discharged up to their maximum rate regardless of the battery cycle or the depth of discharge. Local information must therefore be used in addition to the state of the dc bus to help optimize charging and discharging operations. By using a supervisory controller to dynamically adjust the charge and discharge limits based on the state of the battery bank, the lifetime of the batteries may be extended. For simplicity though, optimal cycling of a battery bank is not considered in this paper.

\section{Load Interface Converter}

Controlling the load interface converter to exhibit different modes of operation based on the voltage level of the dc bus is not a requirement for the implementation of a control law using DBS. However, it is worth noting that DBS has the potential to include demand side management by incorporating this strategy. Since the voltage level of the dc bus reflects a heavy load or a deficiency in available generation, a demandside management strategy can be implemented by controlling the load interface to limit its output power or shed loads when the dc-bus voltage indicates a generation shortage.

\section{IMPLEMENTATION PROCEDURE}

In a hybrid renewable nanogrid, the renewable sources, storage, and backup generation must be utilized in a prioritized fashion to maximize the use of the renewables. The utilization priority for the sources is defined by a system control law, which consists of a number of operating states. The control law is implemented using DBS by first prioritizing the charge/discharge thresholds for the interface converters. Because DBS relies on the bus voltage level to convey system information, reliable operation of the system according to the system control law is then ensured by calculating each threshold with the aid of a dc load flow. Calculating the thresholds ensures that each converter can accurately infer the current operating state of the system despite the bus voltage propagating unequally throughout the system.

To prioritize the sources that discharge into the system, the sources that take the higher priority operating states are assigned to come online at higher discharge thresholds than the lower priority sources. Since the voltage level of the dc bus decreases as a group of sources is consumed, those sources with the higher discharge thresholds come online first. 


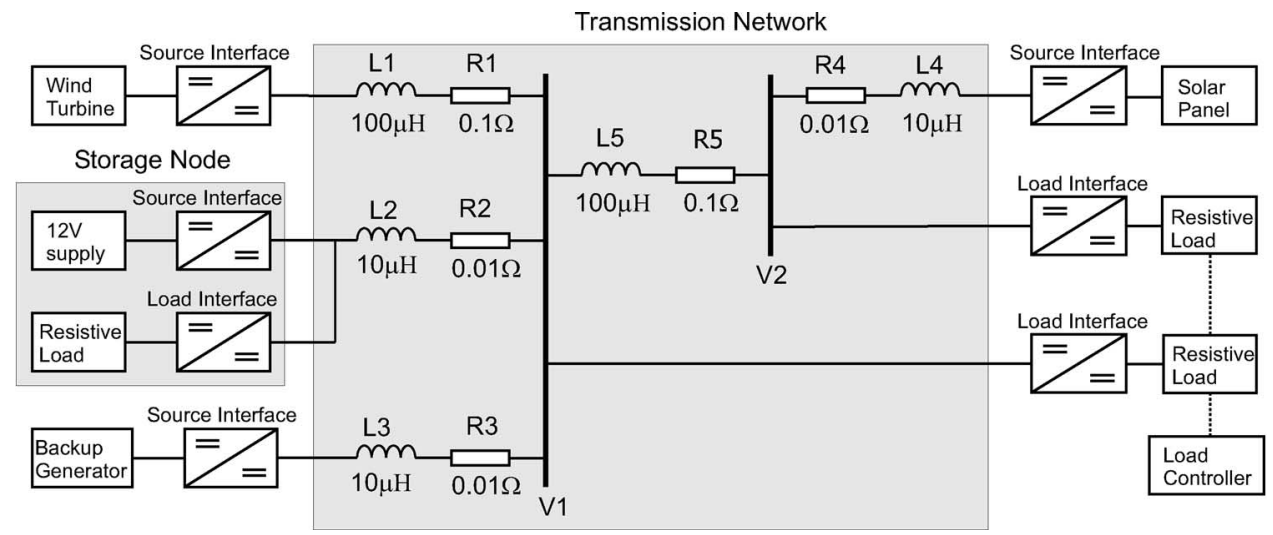

Fig. 6. Experimental system.

Prioritizing the charging of storage nodes is accomplished in the opposite fashion, since charging tends to decrease the bus voltage rather than support it. The storage devices with the highest charging priority are assigned to the lowest charge thresholds.

The thresholds are calculated beginning with the highest threshold. Each successive threshold is calculated to ensure that when the sources assigned to the previous threshold are online, a voltage drop in the system, caused by a transmission line resistance and voltage droop, does not prematurely activate sources assigned to the next threshold. In general, the first discharge threshold $V_{0}$ is set to the nominal operating voltage of the system. Each successive discharge/charge threshold $V_{n}$ is calculated by subtracting the voltage drop and a margin of error from the preceding threshold

$$
V_{n} \leq V_{n-1}-V d_{n}-V_{e}
$$

where

$V_{n-1}$ is the preceding threshold, corresponding to operating state $n-1$;

$V d_{n}$ is the maximum voltage drop that occurs with the system operating in the state $n-1$;

$V_{e} \quad$ is a margin of error that accounts for measurement inaccuracies and voltage ripple on the dc bus.

A dc load flow is used to determine $V d_{n}$. The dc load flow is performed with the system operating in state $n-1$ for all permutations of generation and loading conditions. The worst-case voltage drop is the difference between $V_{n-1}$ and the minimum voltage at the point where any source assigned to threshold $V_{n}$ is connected with the system.

Calculating the thresholds is designed to ensure that the system operates according to the system control law under steady-state operating conditions. This procedure also ensures that the power balance in the system is maintained according to the system control law under transient operating conditions. Provided that the thresholds are calculated to account for the worst case voltage drop in each operating state, the bus voltage level will always indicate the generation required to balance the load. Under a startup transient for example, the DBS may bring an additional source online as the initial surge of current causes the bus voltage to decrease, but the system will settle in its final operating state once the bus voltage has stabilized. It should be
TABLE I

SYSTEM DESIGN

\begin{tabular}{|l|l|}
\hline Source & Vout: $12 \mathrm{~V}$ \\
\hline Source Interface & Vin: $10-15 \mathrm{~V}$ \\
& Vout: $70 \mathrm{~V}$ \\
& Pmax: $100 \mathrm{~W}$ \\
\hline Source Interface Controller & $\begin{array}{l}\text { Bandwidth: } 1 \mathrm{kHz} \\
\text { Voltage Droop: } 0.1 \mathrm{~V} / \mathrm{A}\end{array}$ \\
\hline Load Interface & $\begin{array}{l}\text { Vin: } 60-70 \mathrm{~V} \\
\text { Vout: } 12 \mathrm{~V} \\
\text { Pmax: } 200 \mathrm{~W}\end{array}$ \\
\hline Load Bank & Resolution: $25 \mathrm{~W}$ \\
& Pmax: $200 \mathrm{~W}$ \\
\hline Transmission Network & Resistance: $0.1 \Omega / \mathrm{km}$ \\
& Inductance: $0.1 \mathrm{mH} / \mathrm{km}$ \\
\hline
\end{tabular}

mentioned that short-term transients in the bus voltage in the order of $0.1 \mathrm{~ms}$ have no effect on DBS, since these fluctuations are filtered out by the control circuits for the source/storage interface converters.

\section{EXPERIMENTAL SYSTEM}

Simulation results have shown the feasibility of using the DBS to schedule storage and nonrenewable backup generation in a dc nanogrid [5], [6]. This section presents the design of an experimental nanogrid that is used to demonstrate the practical operation of DBS. The experimental nanogrid is a scaled-down representation of a case study, as shown in Fig. 5.

\section{A. System Characteristics}

The case study is based on a full-scale system that encompasses two generation/load buses located $1 \mathrm{~km}$ apart. The primary source of generation for the system is a wind turbine and photovoltaic array which are sized such that their average output power supplies the average load demand of $2 \mathrm{~kW}$. 


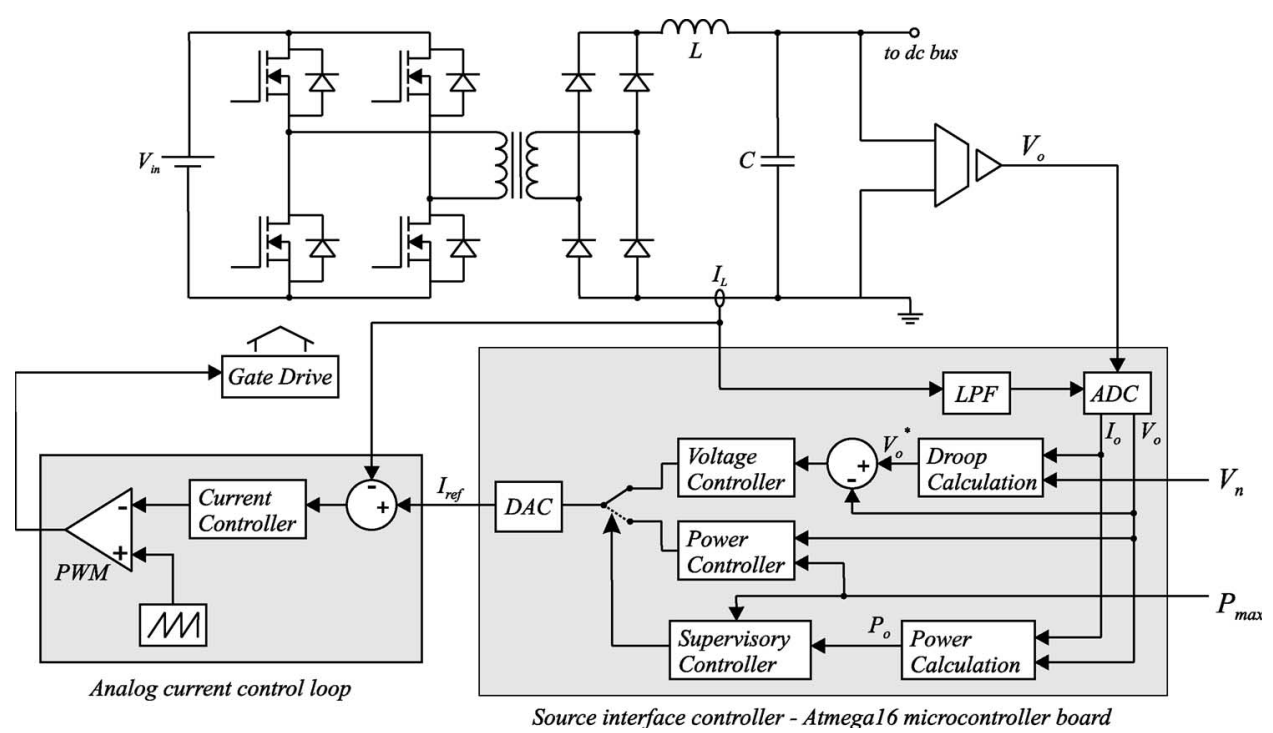

Fig. 7. Source interface and controller implementation.

A battery bank and backup generator maintain a continuous supply of power in the presence of fluctuations in the sources and loads. The parameters of the full-scale system are given as follows: The nominal transmission voltage is $700 \mathrm{~V}$, and the transmission network is an overhead line with a dc resistance of $0.783 \Omega / \mathrm{km}$, an inductance of $0.983 \mathrm{mH} / \mathrm{km}$, and a capacitance of $10 \mathrm{pF} / \mathrm{km}$.

The experimental system is designed to be a scaled replication of the case study. The experimental system, shown in Fig. 6, operates at $70 \mathrm{~V}$, and has an average load demand of $200 \mathrm{~W}$. The key parameters of the system modules are summarized in Table I. It should be noted that the voltage and power ratings of the converter modules are one-tenth the value they would be in the full-scale system.

For simplicity, 12-V laboratory power supplies are used in place of the renewable and nonrenewable sources. Since these supplies do not have an MPP characteristic, the MPP of the renewable sources is accounted for by varying the power limit of the source interface converters.

The source interface is a step-up dc-dc converter that boosts the supply voltage from 12 to $70 \mathrm{~V}$ for connection to the system. A full-bridge hard-switched topology is used, and the control scheme for the converter is shown in Fig. 7.

The source interface controller is implemented using an analog inner current control loop and a digital voltage/power control loop. The inner current loop is based on average current mode control, and the outer control loop is implemented using an Atmel Atmega16 8-bit microcontroller. A supervisory control block selects the mode of operation based on the output power of the converter. When the converter's output power exceeds the MPP of the source, the supervisory controller switches from voltage control to power control mode. The voltage/power control loops are implemented in the microcontroller, and they control the output of the converter by controlling the reference current for the inner loop. A bus voltage conditioning circuit is included in the source interface controller to prevent high-frequency voltage transients above $10 \mathrm{kHz}$ from affecting the operation of the DBS control strategy.
TABLE II

SYSTEM CONTROL LAW

\begin{tabular}{|c|c|c|c|}
\hline State & Renewables & Storage & Generator \\
\hline 1 & Const. voltage & Charge & Off \\
2 & Const. power & Const. voltage & Off \\
3 & Const. power & Const. power & Const. voltage \\
\hline
\end{tabular}

TABLE III

CONTROL-LAW IMPLEMENTATION

\begin{tabular}{|c|c|c|}
\hline Threshold & Description & Value (V) \\
\hline$V_{0}$ & Renewable discharge threshold & 70 \\
$V_{1}$ & Storage charge threshold & 66 \\
$V_{2}$ & Storage discharge threshold & 62 \\
$V_{3}$ & Generator discharge threshold & 58 \\
\hline
\end{tabular}

A bank of incandescent lights is used for each load node. Consisting of six $12-\mathrm{V}$ lights rated at $25 \mathrm{~W}$, each load bank can be controlled in discrete steps of $25 \mathrm{~W}$ up to a peak of $150 \mathrm{~W}$. The load banks exhibit constant power characteristics, because load interface converters connect the load banks to the system.

The construction of the load interface is similar to that of the source interface. The load interface is a full-bridge converter that is controlled using an analog inner current control loop and a microcontroller-based outer voltage control loop.

The storage node is constructed by connecting a source interface and load interface converter in parallel. The load interface converter connects the nanogrid to a resistive load to allow charging action while a source interface converter and $12-\mathrm{V}$ supply allow the storage node to discharge into the nanogrid. It should be noted that the power ratings for charging and discharging are different due to the ratings of the source and load interface converters. 
The transmission line is constructed as a series $R-L$ network, with its parameters derived from the transmission line used in the case study. The parameters of the transmission line are scaled down by a factor of ten, such that the per unit resistance of the line in the experimental system is approximately the same as that of the line in the full-scale 2-kW system. Transmission line capacitance is neglected since this parasitic component is negligible compared to the output capacitance of the source interface converters.

\section{B. System Control Law}

The system control law is designed such that the renewable sources begin discharging first, followed by the storage and backup generator. The storage charges using any excess power from the renewable sources. The system control law is summarized in Table II.

To implement the control law, the three discharge thresholds and single charge threshold are prioritized as shown in Table III, and the voltage level for each threshold is calculated using (5). The worst case voltage drop is subtracted from the preceding threshold to account for the effect of transmission line impedance, and a margin of error of at least $2 \mathrm{~V}$ is used to prevent voltage ripple on the dc bus and measurement inaccuracies from affecting the mechanism of DBS.

It should be noted that the generator has a startup mode of operation to prevent rapid on/off cycling. When the generator comes online, it initially takes the same utilization priority as the renewable sources. Its discharge threshold is increased from $V_{3}$ to $V_{0}$ for a minimum cycle time.

\section{RESULTS}

Disturbances to the loads and sources are applied to the experimental system to demonstrate how the control structure of the interface converters forces changes in the bus voltage that automatically bring other sources online. The results are portrayed in Fig. 8. The load and source powers are varied to force a transition between the operating states of the system.

Fig. 8(a) shows the variations in the load currents, and Fig. 8(b) shows the supply currents from the renewable sources. The current from the storage and diesel nodes required to balance the load demand is shown in Fig. 8(c), while Fig. 8(d) shows the voltage level changes on the nanogrid at bus V1.

Initially, load 1 is $25 \mathrm{~W}$, load 2 is $50 \mathrm{~W}$, and the MPP of the wind turbine and PV array is $70 \mathrm{~W}$. Since the renewable sources are capable of supplying the load, the system operates in state 1 . The storage node extracts the excess power from the renewable sources up to their MPP by regulating the bus voltage at its charge threshold. The resultant charge current is $-1 \mathrm{~A}$.

At $0.5 \mathrm{~s}$, load 1 is increased to $50 \mathrm{~W}$ and load 2 to $75 \mathrm{~W}$. The system still operates in state 1 , since the renewable sources are able to meet the load demand. However, the storage reduces its charging current to $-0.2 \mathrm{~A}$ as the excess renewable power is diminished.

At $1.5 \mathrm{~s}$, load 1 is increased to $75 \mathrm{~W}$ while load 2 remains at $75 \mathrm{~W}$. The system now operates in state 2 since the load exceeds the power available from the renewable sources. The

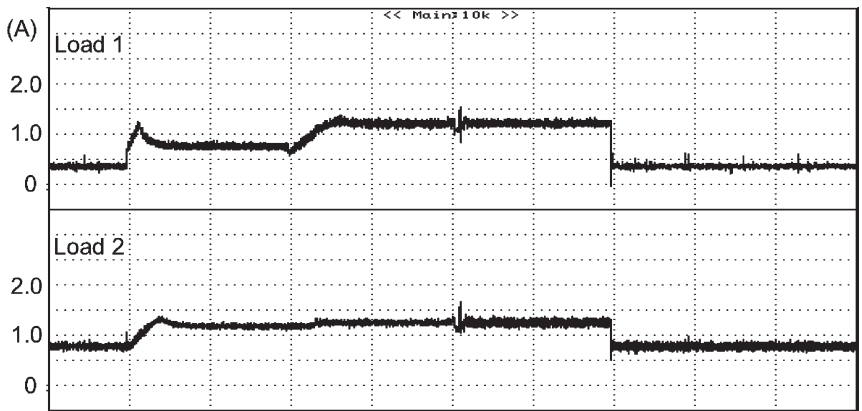

(a)

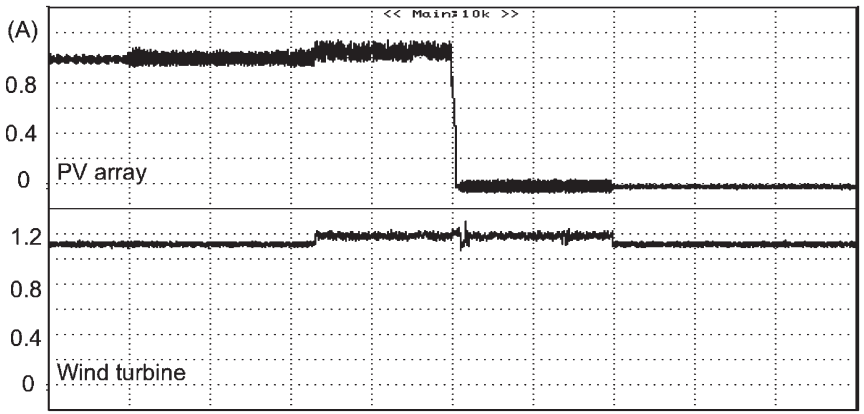

(b)

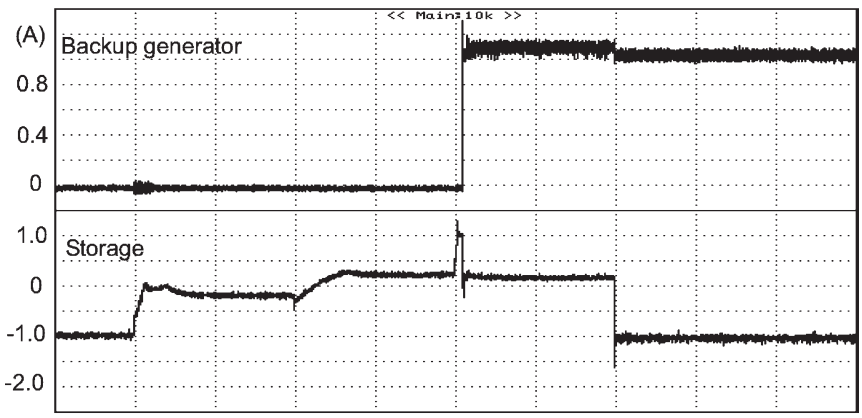

(c)

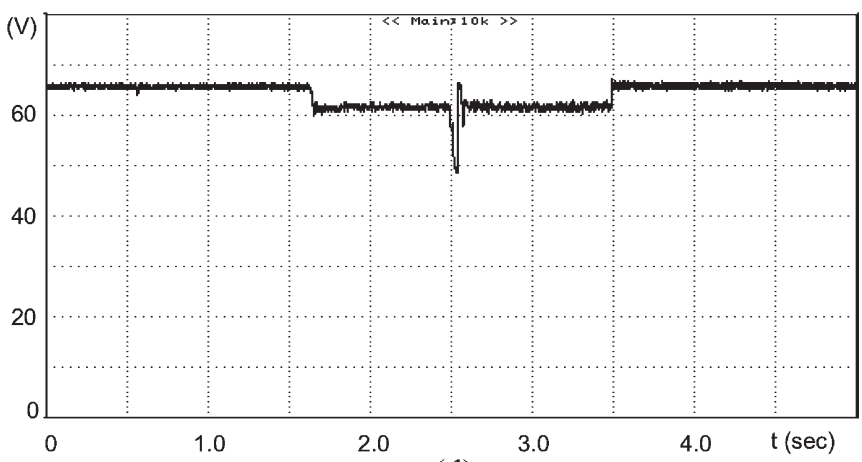

(d)

Fig. 8. Experimental results. (a) Load currents. (b) Renewable source currents. (c) Backup generator/storage current. (d) Voltage at bus V1.

storage node begins discharging, regulating the bus voltage at its discharge threshold in order to maintain the power balance.

At $2.5 \mathrm{~s}$, the PV array is taken offline, reducing the total power from the renewable sources to $70 \mathrm{~W}$. The storage briefly attempts to supply the power balance, but since the load exceeds the maximum output of the wind turbine and storage, these converters operate in power limiting mode, causing the bus voltage to sharply decrease below the discharge threshold of the backup generator. The diesel generator therefore begins discharging, 
taking the same priority as the renewable sources, while the storage remains discharging in order to balance the load.

At $3.5 \mathrm{~s}$, the loads are returned to their initial operating conditions. The backup generator remains online since its minimum cycle time has not elapsed, and the storage node charges from the excess power available from the generator and the wind turbine.

The results have shown that the DBS successfully allows the system to operate according to the system control law based on voltage level changes on the dc bus. Disturbances applied to the system introduced deviations on the dc bus that automatically changed the storage and backup generator's mode of operation to maintain the power balance in the system.

\section{DISCUSSION}

Although the results have verified the feasibility of DBS, several issues must be addressed in order for DBS to operate successfully in a practical system. In practice, the startup time of the backup generator is likely to be in the order of seconds. Therefore, a means of maintaining the power balance in the system while the backup generator is starting is needed. Shortterm storage may be used for this purpose.

Being a system based on power-electronic converters, highfrequency stability is also a major issue. The load interface converters exhibit a negative input impedance at low frequencies due to their constant power characteristics. Since the sources have a higher output impedance at low frequencies, interaction between the sources and loads can result in performance degradation or even instability in the system. Although this issue must be addressed in a practical system, this paper does not attempt to examine this issue since methods of dealing with this problem have been proposed [9], [10].

\section{CONCLUSION}

DBS is a method of scheduling the sources in a powerelectronic-based dc nanogrid without the use of a central controller. DBS is made possible by independently controlling each source and storage interface converter to act as a constant voltage source with a constant power limit. Changes in the load and generation available create voltage-level changes on the dc bus as the converters switch between constant voltage and constant power operation. The dc bus therefore acts as a communication link between the sources, allowing a system control law to be implemented through a local control of each node. Experimental results obtained from a prototype system have demonstrated the operation of this control strategy.

\section{REFERENCES}

[1] R. H. Lasseter, "Microgrids," in Proc. IEEE Power Eng. Soc. Winter Meeting, 2002, vol. 1, pp. 305-308.

[2] J. Bryan, R. Duke, and S. Round, "Distributed generation-Nanogrid transmission and control options," in Proc. Int. Power Eng. Conf., Nov. 2003, vol. 1, pp. 341-346.

[3] W. Dalbon, M. Roscia, and D. Zaninelli, "Hybrid photovoltaic system control for enhancing sustainable energy," in Proc. IEEE Power Eng. Soc. Summer Meeting, 2002, vol. 1, pp. 134-139.

[4] R. Chedid and S. Rahman, "Unit sizing and control of hybrid wind-solar power systems," IEEE Trans. Energy Convers., vol. 12, no. 1, pp. 79-85, Mar. 1997.
[5] J. Bryan, R. Duke, and S. Round, "Decentralised control of a nanogrid," in Proc. Australas. Univ. Power Eng. Conf., Christchurch, New Zealand, Sep. 2003. CDROM.

[6] - "Decentralized generator scheduling in a nanogrid using dc bus signaling," in Proc. IEEE Power Eng. Soc. Summer Meeting, Jun. 2004, pp. 977-982, CDROM.

[7] B. K. Johnson and R. Lasseter, "An industrial power distribution system featuring ups properties," in Proc. IEEE Power Electron. Spec. Conf., Jun. 1993, pp. 759-765.

[8] W. Tang and R. H. Lasseter, "An lvdc industrial power supply system without central control unit," in Proc. IEEE Power Electron. Spec. Conf., 2000, vol. 2, pp. 979-984.

[9] X. Feng, J. Liu, and F. C. Lee, "Impedance specifications for stable dc distributed power systems," IEEE Trans. Power Electron., vol. 17, no. 2, pp. 157-162, Mar. 2002.

[10] G. S. Thandi, R. Zhang, K. Xing, F. C. Lee, and D. Boroyevich, "Modeling, control and stability analysis of a pebb based dc dps," IEEE Trans. Power Del., vol. 14, no. 2, pp. 497-505, Apr. 1999.

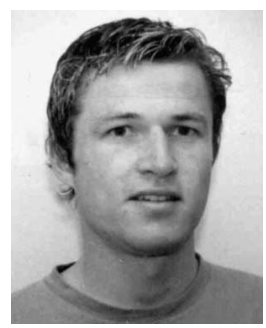

John Schönberger (S’01) received the B.E. (Hons) and Ph.D. degrees from the University of Canterbury, Christchurch, New Zealand, in 2002 and 2006, respectively.

In April 2006, he joined the Power Electronic Systems Laboratory, Swiss Federal Institute of Technology, Zürich, Switzerland, as a Postdoctoral Researcher. His areas of interest include renewable energy systems and the control of distributed $\mathrm{dc}-\mathrm{dc}$ converter-based systems.

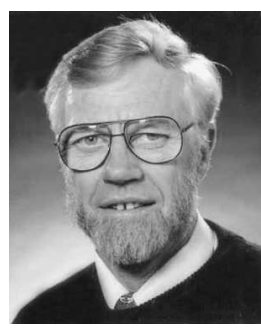

Richard Duke (S'75-M'79) received the Ph.D degree from the University of Canterbury, Christchurch, New Zealand, in 1979.

$\mathrm{He}$ is currently an Associate Professor with the Department of Electrical and Computer Engineering at the University of Canterbury. From 1995 to 2003, he was the Head of Department. His research interests include power electronics and power system harmonics.

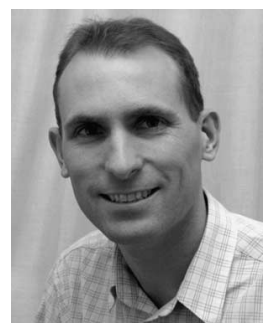

Simon D. Round (S'87-M'89-SM'01) received the B.E. (Hons) and Ph.D. degrees from the University of Canterbury, Christchurch, New Zealand, in 1989 and 1993, respectively.

From 1992 to 1995, he held positions of a Research Associate with the Department of Electrical Engineering at the University of Minnesota and a Research Fellow with the Norwegian Institute of Technology, Trondheim, Norway. From 1995 to 2003, he was a Lecturer/Senior Lecturer (Assistant/Associate Professor) with the Department of Electrical and Electronic Engineering at the University of Canterbury. Recently, he has worked as a power electronic Consultant with Vectek Electronics, where he developed a "state-of-the-art" digital controller for high-power inverter systems. In September 2004, he joined the Power Electronic Systems Laboratory at the Swiss Federal Institute of Technology, Zürich, Switzerland, as a Senior Research Associate. His research interests are in the area of grid-connected systems and digital control of power electronic systems. 